\title{
ESTUDIO COMPARATIVO SOBRE MODELOS DE TURBULENCIA EN FLUJO DE REFRIGERANTES CON BAJO GWP EN EL INTERIOR DE MICROCANALES
}

Eduardo Miranda, Daniel Sempértegui-Tapia y Cristian Chávez

\section{RESUMEN}

Este artículo se trata de una evaluación de modelos de turbulencia para la predicción de la pérdida de presión y el coeficiente de transferencia de calor durante el flujo de refrigerantes con bajo GWP en el interior de microcanales. En este estudio, características de mecánica de fluidos y transferencia de calor se simulan incorporando condiciones de flujo turbulento en un microcanal circular de $1.1 \mathrm{~mm}$ de diámetro. Los modelos de turbulencia con las formulaciones $k$ $\varepsilon$ y $k-\omega$ son evaluados para números de Reynolds hasta 10000 y son implementados computacionalmente en el software OpenFoam. Los perfiles de velocidad, factores de fricción y números de Nusselt son calculados y analizados en función de variables paramétricas. Los resultados numéricos son validados con métodos de predicción analíticos y con datos experimentales medidos en trabajos previos para flujos de R134a, R1234fy, R1234ze(E) y R600a. La variación de la caída de presión y coeficiente de transferencia de calor convectivo son analizados de acuerdo al efecto al tipo de fluido y al modelo de turbulencia empleado. Los resultados indican que los modelos describen cualitativamente el comportamiento del flujo y la transferencia de calor en condiciones de turbulencia. Cuantitativamente, los modelos Low-Re $k-\varepsilon, k-\omega$ estándar y $k-\omega$ SST presentan mejores predicciones para las variables de interés. Por otro lado, el modelo Low-Re $k-\varepsilon$ presenta el menor error medio absoluto.

Palabras Clave: Turbulencia, Microcanales, Numérico, Caída de Presión, Transferencia de Calor.

DOI: 10.23881/idupbo.021.1-6i 